of the structures of vitamins $B_{1}$ and $B_{2}$. Vitamin $\mathrm{B}_{1}$ has the formula $\mathrm{C}_{12} \mathrm{H}_{18} \mathrm{O}_{2} \mathrm{~N}_{4} \mathrm{~S}$. There appear to be two ring systems present; one a glyoxaline or pyrimidine, the other a pyrrole containing a substituent sulphur. Interest in vitamin $\mathrm{B}_{2}$ has been greatly stimulated by the recent discovery that the flavins, which are yellow water-soluble dyes present in both animal and vegetable sources, possess intense vitamin $B_{2}$ activity. The flavins are derivatives of alloxazine, and a substance stated to be identical with lactoflavin of milk whey has already been synthesised. It possesses the complex ring systems shown. So discovery<smiles></smiles>

One of ring systems of
vitamin $B$

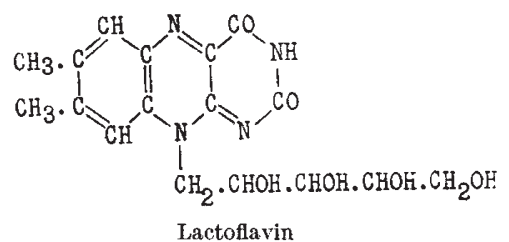

Lactoflavin marches onwards! Who shall say what additional structural formulæ will be recorded in a similar survey after the lapse of another quarter of a century?

\title{
Diet and Disease
}

By Prof. Stuart J. Cowerr, Professor of Dietetics, St. Thomas's Hospital Medical School, London

$\mathrm{T}$ HE twenty-five years of His Majesty's reign which are now being celebrated correspond remarkably closely with the establishment of a new era in the science of nutrition. At the opening of the twentieth century, attention was being focused on the quantitative relations of the energy exchanges of the body and on the metabolism within the body of the proteins, fats and carbohydrates of the food. The physiologists and chemists working at these problems were making most valuable contributions to the body of knowledge concerning the processes of nutrition, but such contributions were for the most part not of such a nature as to afford obvious clues either to the origin of or to the treatment of disease. The second decade of the twentieth century witnessed the rapid development of the view that the adequate nutrition of an animal depended on the presence in its food of hitherto unsuspected elements. The absence of such essential elements from a diet was proved to result regularly in the appearance of predictable signs of disease, and the fundamentally new idea of deficiency diseases became gradually established in current medical teaching.

Before mentioning any of the effects which this new conception of nutrition has had on the problems of the prevention and treatment of disease, it will be useful to hark back to the 'pre-Georgian' era to review the current teaching of the medical profession regarding the relation of diet to disease. The fact that faulty diets are often the direct or indirect cause of disease was of course fully recognised, as it had been for many centuries. But there was little precise knowledge available to enable definite diseases to be ascribed to specific dietetic errors. Over-eating was regarded as predisposing to many gastro-intestinal diseases, gout and raised blood pressure, and under-eating was considered to render the body more liable to invasion by harmful bacteria. The idea of lack of balance between the various classes of foodstuffs, for example, relative excess or deficiency of protein, carbohydrate or fat was looked upon as at least an important contributory cause of disease. In the case of scurvy, it was already taught that the absence from the diet of some principle which was present in fresh foods but not in stale foods contributed largely to the production of the disorder. Otherwise the production of disease by faulty diet was largely related to the presence of toxins, pathogenic bacteria or living parasites in food which had become accidentally contaminated.

With regard to the practical dietetic management of diseases now known to be due to specific dietetic faults, the degree of divergence between the methods of twenty-five years ago and of the present day is distinctly less than would have been expected from a consideration of the knowledge available then and now. Specific remedies in medicine have again and again been discovered empirically, and this is true in the realm of dietetics. The treatment recommended twentyfive years ago by at least some enlightened authorities for many of the diseases now spoken of as deficiency diseases would prove satisfactory enough to-day, although such treatment was based on no actual knowledge of the dietetic factors involved. Scurvy was treated by giving fresh fruit and fresh vegetables, rickets and osteomalacia by giving cod liver oil and milk, beriberi by increasing the 'nitrogenous' constituents and diminishing the carbohydrate of the diet-some individuals were even claiming that it could be prevented by adding rice polishings to the diet of highly polished rice which was usually eaten in districts where this disease occurred-and finally pellagra was 
to be treated by cutting maize out of the diet.

This list of diseases comprises most of those commonly regarded to-day as vitamin deficiency diseases, and there are not lacking critics of modern nutritional research who profess to be unimpressed by its practical value in clinical medicine because many of its obvious applications had been forestalled by empirical methods of treatment. Such arguments fail to recognise the frequency with which valuable methods of treatment fall into disuse or are replaced at least temporarily by worthless imitations when little or nothing is known of the physiological action of the agents effective in alleviating the symptoms of disease. Thus the value of fresh lemon juice in curing scurvy was known in the eighteenth century, but was forgotten for long periods, and far inferior therapeutic agents were sanctioned by high authorities as late as the present century. Similarly the value of cod liver oil for the cure of rickets had been appreciated for many years before the discovery of vitamins, but this did not prevent the subsequent recommendation of inert vegetable fats as satisfactory substitutes.

It is not argued that empirical treatment has no stable foundation in therapeutics, for clinical medicine still makes use of many old-established therapeutic measures which have as yet no scientific basis. But it is no less certain that the scientific demonstration of a definite cause for a disease offers the best chance for the discovery of satisfactory preventive and curative treatment, and when the cause is proved to be a comparatively simple deficiency in the diet, there should be no excuse whatever for allowing such knowledge to sink into oblivion.

It is now proposed to show how the conception of food deficiencies as a cause of disease has developed during the past twenty-five years. The great stimulus to the remarkable activity shown in this field of medical investigation during this period was undoubtedly the discovery of the vitamins, but the success rapidly attained by those engaged in studying the effects of vitamin deficiencies encouraged the investigation of the effects of other deficiencies, for example, deficiency in the mineral components of the diet, with the result that in such fields also many observations of the greatest importance have been made which have already proved invaluable alike in clinical and in veterinary medicine.

The period of nutritional research which we are surveying had already been heralded by isolated suggestions regarding the importance of dietetic factors other than proteins, fats, carbohydrates and minerals salts for the maintenance of health. Already in 1897, Eijkman had published his experiments on the production of beriberi, which led ultimately to the discovery of the antineuritic vitamin. Hopkins had stated in 1906 his conviction that scurvy, rickets and probably other states of ill-health were caused by unknown dietetic errors the nature of which was bound up with a defective supply of obscure food components. In 1907 Holst and Frölich paved the way for the identification of the antiscorbutic vitamin by producing experimental scurvy in guinea pigs. But it was not until Hopkins had demonstrated in 1912 the fundamental importance of 'accessory factors' in the diet for securing normal nutrition that the idea of specific food deficiencies as a cause of disease began to gain any general acceptance.

It is common knowledge that, since Hopkins's original announcement, the number of accessory food factors or vitamins generally recognised as being concerned in animal nutrition has been steadily increasing, though not all of them have been shown to be concerned in the production of human deficiency diseases. By the end of the first decade of King George's reign, overwhelming evidence had been produced to show that beriberi was produced by deficiency of the water-soluble antineuritic vitamin, xerophthalmia by deficiency of a fat-soluble vitamin, rickets by deficiency of the same or a similar vitamin and scurvy by deficiency of the water-soluble antiscorbutic vita$\min$. It is not possible to trace in detail subsequent investigations, which have led on one hand to the chemical identification of many of the vitamins and on the other to some understanding of their physiological action. It must suffice to point out how such knowledge has been applied to the prevention and treatment of disease.

In countries such as Great Britain, frank vitamin-deficiency diseases, with the single exception of rickets, are uncommon, but there is increasing evidence that partial deficiencies of vitamins, particularly during the period of growth, are often responsible for sub-optimal physical development, inperfections in the structure of bodily organs and tissues, lowered resistance to certain infective diseases and many vague subjective and objective symptoms of ill-health. It is almost certain, for example, that mild degrees of skeletal deformity caused by faulty feeding in childhood help to raise maternal mortality in childbirth by increasing the mechanical difficulties of labour. The low resistance of the teeth of large sections of our population to decay, with its many sequelæ of chronic disease and ill-health, is due in large part to faulty development of the teeth during the early years of life brought about by dietetic faults, of which vitamin $\mathrm{D}$ deficiency is the most prominent. Similarly it has been shown that, in dogs at least, the tendency to the development 
of pyorrhcea alveolaris in adult life is largely determined by the supply of vitamin A which was available during the period of growth. These few illustrations must suffice to indicate that the original conception of vitamin-deficiency diseases has extended its boundaries to include a variety of diseases attributable to past dietary deficiencies, preventable by suitable feeding during the period of growth.

Similar results have been achieved during the period under review by the study of specific mineral deficiency diseases. Prominent among those mineral elements deficiency of which in the diet may lead to recognisable symptoms of disease are iron, copper, iodine, phosphorus, calcium and magnesium. Lack of sufficient iron in the food has been shown to result frequently in the development of anæmia. Such 'nutritional' anæmia is particularly common in infants, and is accounted for by the fact that milk is a poor source of iron. The recognition of this form of anæmia has proved to be of considerable practical importance, because it is often associated with an increased susceptibility to many of the common complaints of infancy and can be corrected with great ease. The enormous amount of work that has lately been carried out on the relation between iodine deficiency and the development of goitre has not yet completely solved the problem of the causes of thyroid enlargement, but it has certainly provided very successful methods of wholesale prophylaxis in districts where goitre is endemic. Lastly may be mentioned the revolution in the cattle-rearing industry of South Africa which has followed the discovery of phosphorus deficiency as the immediate or ultimate cause of serious losses in this branch of agriculture.

There can be no question that already the modern conception of nutrition has produced practical results of immense significance in both preventive and curative clinical and veterinary medicine. The results are not confined to the prophylaxis and treatment of diseases which arise as the result of actual deficiencies in the food supplied. They have already been extended to include the treatment of diseases in which defects in the absorption or utilisation of particular food elements rather than faulty diets are responsible for the development of co-existing signs of deficiency diseases. The term 'secondary deficiency disease' is now being used to distinguish this particular class of nutritional disorder. In this group might well be placed that once fatal malady pernicious anæmia, which the brilliant researches of the past decade have shown to be amenable to simple dietetic treatment, although there is no evidence that dietetic errors play any part in its causation. As more and more precise information is gained concerning the intimate processes of metabolism within the body, there should be increasing opportunities of preventing and successfully treating disease by adjusting the diet to influence those metabolic processes which may produce the symptoms of disease when they deviate from their normal course. This has long been one of the aims of clinical medicine; its ultimate realisation has surely been brought one stage nearer fulfilment by the nutritional investigations of the past twenty-five years.

\section{Viruses as the Cause of Disease}

By Dr. Joseph A. Arkwright, F.r.s., Lister Institute of Preventive Medicine, London

$\mathrm{A}^{\mathrm{T}}$ the end of the nineteenth century, a new category of infective agents was discovered which are now classed as viruses, in the modern sense of the word. The chief property which unites them, and by which they have been distinguished from previously known minute parasites, is the extremely small size of their component particles, since these are smaller than bacteria, and many of them are not visible even with the highest powers of the microscope. Until quite recently, it was customary to speak of all viruses as invisible, but in some cases the minute granules of which the virus appears to consist can be clearly seen, when stained, by direct microscopic observation; but in most cases they cannot be distinguished by their shape, but only by their uniformity, numbers and their source in special parts of the diseased tissues. The recent investigations by
Barnard with the ultra-microscope and photomicrography by ultra-violet light have added to our knowledge of their size and form.

A special feature by which the invasion of the cells of the host by many viruses can be recognised is the occurrence of 'cell inclusion bodies'. These forms, of which there may be one or more in a single cell, vary in size and may be larger than the nucleus. Opinion as to their nature has undergono various vicissitudes. After their discovery, when they were at first hailed as protozoal parasites, they were for long regarded as merely reaction products of the cell protoplasm to the presence of the virus, a position now favoured for the 'inclusion bodies' associated with virus diseases of plants. More recently it has been shown that in some virus diseases of animals these inclusion bodies' consist of masses of the minute filterable 